\title{
Ouabain-insensitive K-Adenosine Triphosphatase in Distal Nephron Segments of the Rabbit
}

\author{
Lal C. Garg and Neelam Narang \\ Department of Pharmacology and Therapeutics, University of Florida College of Medicine, Gainesville, Florida 32610
}

\begin{abstract}
An electrogenic H-ATPase sensitive to inhibition by $\boldsymbol{N}$-ethylmaleimide has been reported to be present in renal distal tubules. In contrast to another H-ATPase (gastric H-K-ATPase), the renal enzyme is not stimulated by $\mathrm{K}^{+}$and is not inhibited by vanadate. However, our preliminary observations indicated that a K-stimulated ATPase (K-ATPase) sensitive to inhibition by vanadate is present in renal medullary collecting duct (MCD). To localize and further characterize this renal tubular K-ATPase, we measured K-ATPase activity in eight specific segments of the rabbit nephron. K-ATPase activity was the difference in ATPase activity in the presence and absence of $\mathrm{KCl}$ but in the presence of ouabain (to inhibit $\mathrm{Na}-\mathrm{K}$ ATPase). ATPase activity was determined by a fluorometric microassay in which ATP hydrolysis is coupled to the oxidation of NADH. There was a significant K-ATPase activity (expressed as $\mathrm{pmol} \cdot \mathrm{min}^{-1} \cdot \mathrm{mm}^{-1}$ ) in the connecting tubule (CNT, 17.0 \pm 3.3$)$, cortical collecting duct (CCD, 6.6 \pm 0.7$)$, and MCD (8.8 \pm 1.7$)$, but not in the proximal segments and the thick ascending limbs. The renal tubular K-ATPase was not only inhibited by vanadate but also by omeprazole and SCH 28080 (relatively specific inhibitors of gastric H-K-ATPase). It is concluded that K-ATPase present in the CNT, CCD, and MCD has some properties in common with gastric H-K-ATPase. However, the physiological role of K-ATPase in the distal nephron segments remains to be elucidated.
\end{abstract}

\section{Introduction}

The renal medullary collecting duct $(\mathrm{MCD})^{1}$ has been shown to secrete $\mathrm{H}^{+}$and absorb $\mathrm{K}^{+}(1,2)$. The secretion of $\mathrm{H}^{+}$in this and other collecting duct segments is attributed to an electrogenic H-ATPase that is sensitive to inhibition by $N$-ethylmaleimide (NEM) but not to vanadate (2-6). During our recent studies on NEM-sensitive ATPase in distal nephron segments

Address correspondence and reprint requests to Dr. Garg, Dept. of Pharmacology, College of Medicine, University of Florida, Box J-267 J. Hillis Miller Health Center, Gainesville, FL 32610.

Received for publication 20 July 1987 and in revised form 9 November 1987.

1. Abbreviations used in this paper: $\mathrm{CCD}$, cortical collecting duct; CNT, connecting tubule; CTAL, cortical thick ascending limb; DCT, distal convoluted tubule; K-ATPase, K-stimulated ATPase; MCD, medullary collecting duct; MTAL, medullary thick ascending limb; NEM, $N$-ethylmaleimide; PCT, proximal convoluted tubule; PST, proximal straight tubule.

J. Clin. Invest.

(C) The American Society for Clinical Investigation, Inc.

0021-9738/88/04/1204/05 \$2.00

Volume 81, April 1988, 1204-1208
(5), we observed that a vanadate-sensitive ATPase was present in the MCD but not in the medullary thick ascending limb (MTAL) (7). Vanadate is known to inhibit the $E_{1}-E_{2}$ type of ATPases that include Na-K-ATPase, Ca-ATPase, and H-KATPase. In our ATPase assay system, we used ouabain to completely inhibit Na-K-ATPase. In addition, we used EDTA in our incubation medium (but no $\mathrm{Ca}^{++}$) to exclude the activity of Ca-ATPase. To determine if vanadate-sensitive ATPase in the MCD is similar to gastric H-K-ATPase, we determined K-stimulated ATPase (K-ATPase) activity in the MCD and other nephron segments of the rabbit. Our preliminary observations indicated that ouabain-insensitive K-ATPase was present not only in the MCD but also in the cortical collecting duct (CCD) and connecting tubule (CNT), but not in the proximal tubules and the loop of Henle (8). The gastric H-KATPase is not only stimulated by $\mathrm{K}^{+}$and inhibited by a nonspecific inhibitor vanadate but is also inhibited by some relatively specific inhibitors such as omeprazole and $\mathrm{SCH}$ 28080 (9-12).

The purpose of the present study was to $(a)$ localize KATPase along the rabbit nephron, and $(b)$ to further characterize renal tubular K-ATPase regarding its dependence on the concentration of $\mathrm{K}^{+}$and its inhibition by omeprazole and SCH 28080. Therefore, we measured K-ATPase activity in eight types of microdissected segments from the rabbit nephron. The segments examined were: the proximal convoluted tubule (PCT), proximal straight tubule (PST), MTAL, cortical thick ascending limb (CTAL), distal convoluted tubule (DCT), CNT, CCD, and MCD. In addition, the dependency of K-ATPase on the concentration of $\mathrm{K}^{+}$was determined in the CCD, and the sensitivity of K-ATPase activity to inhibition by vanadate, omeprazole, and SCH 28028 was determined in the CNT, CCD, and MCD.

\section{Methods}

Animals and microdissection of nephron segments. Male New Zealand white rabbits weighing 1-1.5 kg were fed a regular rabbit chow (Ralston-Purina Co., St. Louis, MO) ad lib. The animals had free access to drinking water. The method for microdissection of rabbit nephron segments was the same as described previously (13). In summary, the rabbits were killed by decapitation and the left kidney was excised and perfused with a chilled buffer containing collagenase. The perfusion buffer contained (in millimolars): $\mathrm{NaCl}, 136 ; \mathrm{KCl}, 3 ; \mathrm{K}_{2} \mathrm{HPO}_{4}, 1$; $\mathrm{MgSO}_{4}, 1.2 ; \mathrm{CaCl}_{2}, 2 ; \mathrm{Na}$ lactate, 4 ; $\mathrm{Na}$ citrate, 1; L-alanine, 6; and glucose, 5.5. The collagenase when present was $0.1 \%$ (type 1,140 $\mathrm{U} / \mathrm{mg}$, Sigma Chemical Co., St. Louis, MO). Tangential and sagittal slices were taken from the perfused kidney and incubated in the perfusion buffer (containing collagenase), which was bubbled with $100 \% \mathrm{O}_{2}$, for $30-80 \mathrm{~min}$ at $37^{\circ} \mathrm{C}$. Individual nephron segments were dissected in cold perfusion buffer (which did not contain $\mathrm{KCl}, \mathrm{CaCl}_{2}$, and collagenase) under the stereomicroscope. The nephron segments microdissected were: PCT next to the glomerulus; PST next to the thin descending limb; MTAL next to the thin ascending limb; CTAL adjacent to macula densa; DCT next to macula densa area; CNT, the granular part 
of the late distal tubule; $\mathrm{CCD}$ from the medullary ray; and MCD from the inner stripe of the outer medulla. The length of each segment was measured by an eyepiece micrometer.

ATPase assay. The method for determination of K-ATPase activity is a modification of our assay used for determination of Na-K-ATPase activity in microdissected nephron segments (13). The method is based on the hydrolysis of ATP to ADP, which is coupled to oxidation of NADH (14) as follows:

ATP $\underset{\text { ATPase }}{\longrightarrow}$ ADP + inorganic phosphate,

$\mathrm{ADP}+$ phosphoenolpyruvate $\underset{\substack{\text { pyruvate } \\ \text { kinase }}}{\longrightarrow} \mathrm{ATP}+$ pyruvate

pyruvate + NADH $\underset{\text { dehydrogenase }}{\stackrel{\text { lactate }}{\longrightarrow}} \mathrm{NAD}+$ lactate.

All three reactions were performed in $300 \mu$ l of imidazole buffer in the same vial. The final concentration of the incubation medium $(\mathrm{pH}$ 7.4) was: $25 \mathrm{mM}$ imidazole, $10 \mathrm{mM} \mathrm{MgCl}, 2.5 \mathrm{mM}$ sodium azide, 1 mM ouabain, $0.6 \mathrm{mM}$ phosphoenolpyruvate, $1.1 \mathrm{mM} \mathrm{Na}_{2}$ ATP, 0.017 $\mathrm{mM} \mathrm{NADH}, 0.33 \mathrm{mM}$ EDTA, $3.2 \mathrm{U} / \mathrm{ml}$ of pyruvate kinase, and 4.1 $\mathrm{U} / \mathrm{ml}$ of lactate dehydrogenase. Ouabain was added to inhibit Na-KATPase, which is a major fraction of total ATPases in the renal tubules (13). Sodium azide was added to decrease the background by inhibiting mitochondrial and other nonspecific ATPases present in the renal tubules (15). For determination of K-ATPase, one-half of the number of samples were incubated in the imidazole buffer, which contained 2.5 $\mathrm{mM} \mathrm{KCl}$, and the other half were incubated in the buffer without any $\mathrm{KCl}$. The difference between the ATPase activity in the presence and absence of $\mathrm{KCl}$ is called $\mathrm{K}-\mathrm{ATP}$ ase activity.

Preparation of the nephron segments for enzyme assay. We and others (13) have observed that to get the maximal ATPase activity in the rabbit nephron segments, the tissue must be given an osmotic and temperature shock. The increase in ATPase activity by this treatment is probably due to an increase in permeability of the cells to ATP and other reactants. Therefore, the microdissected nephron segments were transferred individually to a test tube in $100 \mu \mathrm{l}$ of a hypotonic solution ( $1 \mathrm{mM}$ imidazole, $1 \mathrm{mM} \mathrm{MgCl}, 1 \mathrm{mM}$ EDTA, and $0.1 \%$ bovine serum albumin). The tubes containing the samples were frozen in a mixture of dry ice and acetone. After $\mathbf{4 0} \mathrm{min}$, the frozen samples were thawed. In one-half the number of samples, $100 \mu l$ of imidazole buffer (pH 7.4) (containing $25 \mathrm{mM}$ imidazole, $30 \mathrm{mM} \mathrm{MgCl}$, $7.5 \mathrm{mM}$ sodium azide, and $3 \mathrm{mM}$ ouabain) without $\mathrm{KCl}$ was added to each sample. In the other half of the samples, $100 \mu$ l of imidazole buffer (of same composition and $\mathrm{pH}$ as above) with $2.5 \mathrm{~m} \mathrm{KCl}$ was added to each sample.

Incubation of microdissected nephron segments for ATP hydrolysis. The reaction (ATP hydrolysis) was started by addition of $100 \mu \mathrm{l}$ of a starting buffer (pH 7.4) (containing $50 \mathrm{mM}$ imidazole, $3.3 \mathrm{mM} \mathrm{Na}$ ATP, $1.8 \mathrm{mM}$ phosphoenol pyruvate, $0.05 \mathrm{mM}$ NADH, $9.6 \mathrm{U} / \mathrm{ml}$ of pyruvate kinase, and $12.3 \mathrm{U} / \mathrm{ml}$ of lactate dehydrogenase) to each sample and incubation of the sample in a shaking water bath at $37^{\circ} \mathrm{C}$. After $30 \mathrm{~min}$ of incubation, $\sim 100 \mu$ l of the incubated solution was placed in a microcuvette, and NADH fluorescence was measured at $465 \mathrm{nM}$ in a modified Turner fluorometer (model 111, with an excitation wavelength set at $345 \mathrm{nM}$ ).

Standard curve and calculation of ATPase activity. We determined the relationship between the concentration of ADP and the fluorescence intensity of NADH in our assay system (in the absence of nephron segments). The decrease in the fluorescence was proportional to ADP concentration. Therefore, ADP generated in this assay system was determined by a decrease in NADH fluorescence, which we did in the case of Na-K-ATPase and NEM-sensitive ATPase systems $(5,13)$. All the reactants in this system were in considerable excess and the reaction with $\mathrm{ADP}$ was complete in $<1 \mathrm{~min}$, the only limiting factor being the generation of ADP from ATP by ATPases. Because ADP standard curves were reproducible from day to day, we calculated
ATPase activity from two measurements (one without any ADP and the other with a known amount of $\mathrm{ADP}$ in each experiment $(5,13)$.

Concentration of ATP. We determined change in NADH fluorescence in our assay system without any added ATP and with two concentrations ( 1.1 and $10 \mathrm{mM}$ ) of ATP along with microdissected CCD. There was no decrease in NADH fluorescence in the absence of added ATP, which indicated that there was no significant endogenous ATP (or ATPase activity) in these permeabilized cells. Furthermore, there was no difference in the activities of total ATPase or K-ATPase between the two concentrations ( 1.1 and $10 \mathrm{mM}$ ) of ATP used. Therefore, consistent with our previous method of determination of $\mathrm{Na}-\mathrm{K}$ ATPase and NEM-sensitive ATPase activity $(5,13)$, we used $1.1 \mathrm{mM}$ ATP in all our assays.

Concentration of $\mathrm{K}^{+}$. In some experiments, the concentration of $\mathrm{KCl}$ in the incubation media was varied from 0 to $4 \mathrm{mM}$. To determine the specificity of $\mathrm{K}^{+}$for K-ATPase, we also determined the effect of $0-10 \mathrm{mM} \mathrm{NaCl}$ on stimulation of ATPase activity in the CCD in our assay system.

pH dependency of K-ATPase. To determine the optimum $\mathrm{pH}$ of K-ATPase in our assay system, we determined K-ATPase activity in buffers having $\mathrm{pHs}$ from 7 to 7.8 . Imidazole/ $\mathrm{HCl}$ buffers were used for determination of ATPase activity at $\mathrm{pH} 7.0,7.2,7.4,7.6$, and 7.8. In addition, at $\mathrm{pH} 7.8$, we also used Tris/ $\mathrm{HCl}$ buffer (of the same strength as imidazole $/ \mathrm{HCl}$ ) for determination of ATPase activity.

Use of inhibitors. To determine the sensitivity of K-ATPase to various inhibitors, K-ATPase activity was determined in the presence and absence of $20 \mu \mathrm{M}$ sodium orthovanadate, $100 \mu \mathrm{M}$ omeprazole, and $100 \mu \mathrm{M} \mathrm{SCH} 28080$. Each inhibitor was added (three times the final concentration) to the imidazole buffer that was added to one-half the number of samples after treating the nephron segments with a hypotonic solution (see above the preparation of nephron segments for enzyme assay).

Materials. Omeprazole was a gift from A. B. Hassle, and kindly provided by Dr. Bjorn Wallmark, and compound SCH 28080 was a gift from Schering Corp. kindly provided by Dr. James Kaminski. All other chemicals and enzymes were obtained from Sigma Chemical Co.

Statistical analysis. Results presented in the figures are expressed as mean values \pm SEM from several animals. $K$-ATPase activity in each nephron was compared with zero (Fig. 1) or with the enzyme activity in the presence of inhibitors (Figs. 4 and 5) by the Student's $t$ test. A $P$ value $<0.05$ was considered statistically significant.

\section{Results}

K-ATPase activity in the proximal and loop of Henle segments (PCT, PST, MTAL, CTAL, and DCT) of the rabbit nephron was not significantly different from zero (Fig. 1). On the other hand, there was significant K-ATPase activity in the CNT,

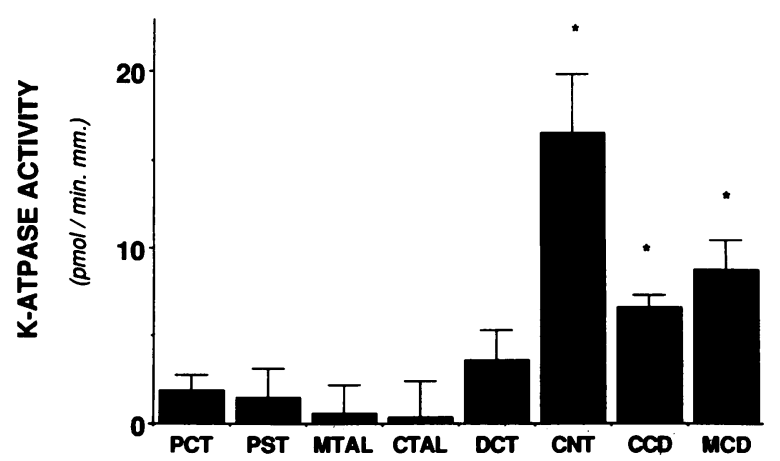

Figure 1. Distribution of K-ATPase activity along the rabbit nephron. Each bar represents mean \pm SEM of four to seven animals. ${ }^{*} P<0.05$ vs. 0 . 


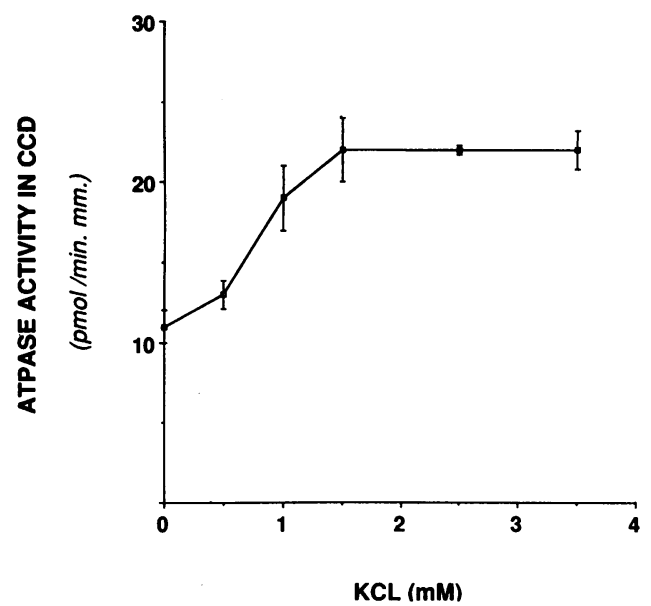

Figure 2. Effect of potassium concentrations on ATPase activity in the CCD. Each point is mean \pm SEM of three to four animals.

CCD, and MCD. K-ATPase activity in the CNT was the highest among these three segments and was almost twice that of the CCD or MCD.

K-ATPase activity in the CCD was dependent on the concentration of $\mathrm{KCl}$ in the incubation media (Fig. 2). The stimulation of ATPase was apparent at $<1.0 \mathrm{mM} \mathrm{KCl}$ and reached its maximum at $1.5 \mathrm{mM} \mathrm{KCl}$. In contrast to $\mathrm{KCl}$, there was no stimulation of ATPase activity in the $\mathrm{CCD}$ with $\mathrm{NaCl}$ (Table I). The dependency of K-ATPase activity in the CNT and MCD on $\mathrm{K}^{+}$concentration in the incubation media was similar to that of the CCD (data not shown).

K-ATPase activity was also dependent on the $\mathrm{pH}$ of the incubation media (Fig. 3). The enzyme activity was maximum at $\mathrm{pH} 7.4$, and the enzyme activity decreased both by increasing and decreasing the $\mathrm{pH}$ of the incubation medium.

$\mathrm{K}-\mathrm{AT}$ Pase activity in the CNT, CCD, and MCD was completely inhibited by vanadate $(20 \mu \mathrm{M})$ and omeprazole (100 $\mu \mathrm{M})$ (Fig. 4). There was no significant effect of vanadate on ATPase activity in our assay system in the absence of $\mathrm{KCl}$. The negative values with omeprazole in the $C C D$ and $M C D$ suggest that omeprazole-sensitive ATPase activity is greater than Kstimulated ATPase activity. This may be due to either some $\mathrm{K}$-ATPase activity even without any $\mathrm{KCl}$ in the medium, or omeprazole may be inhibiting not only K-ATPase but also some other ATPase in these nephron segments. Note that we determined $\mathrm{K}^{+}$(by flame photometry) in a few samples after incubation. There was no detectable quantity of $\mathrm{K}^{+}$in any of

Table I. Comparisons of Effects of $\mathrm{KCl}$ and $\mathrm{NaCl}$ on ATPase Activity in the CCD

\begin{tabular}{cc}
\hline Salts added & ATPase activity in CCD \\
\hline$m M$ & $p m o l \cdot \mathrm{min}^{-1} \cdot \mathrm{mm}^{-1}$ \\
0 & $10.8 \pm 0.2$ \\
$\mathrm{NaCl}(10)$ & $12.0 \pm 2.3$ \\
$\mathrm{KCl}(2.5)$ & $20.0 \pm 2.6$
\end{tabular}

Values are mean $\pm \mathrm{SE}$ of three animals.

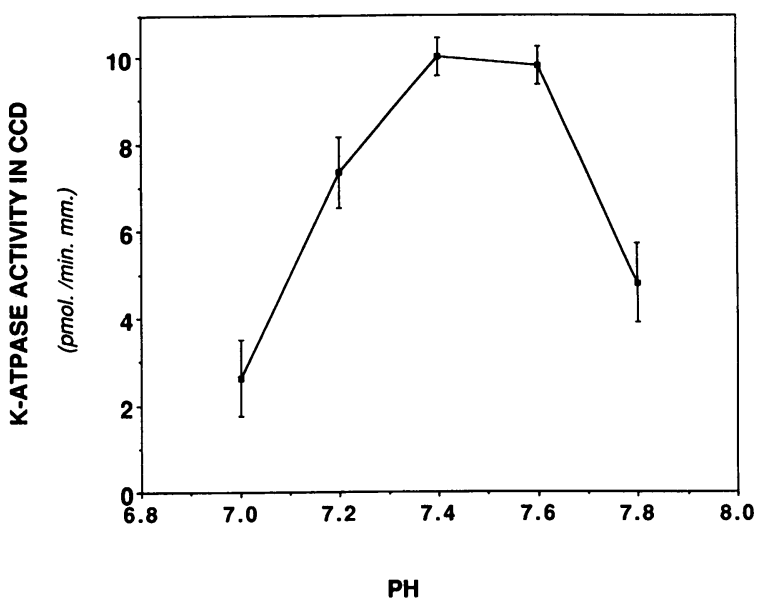

Figure 3. Effect of $\mathrm{pH}$ of the incubation medium on K-ATPase activity in the CCD. Each point is mean \pm SEM of three to four animals.

these samples. K-ATPase activity in the CNT, CCD, and MCD was also inhibited by SCH $28080(100 \mu \mathrm{M})$ (Fig. 5). This compound is much more specific for inhibition of K-ATPase than omeprazole and vanadate (see Discussion).

\section{Discussion}

We have documented the presence of an ouabain-insensitive $\mathrm{K}-\mathrm{ATPase}$ in the CNT, CCD, and MCD of the rabbit nephron. The complete biochemical characteristics and physiological role of this K-ATPase in the kidney is not known at the present time. However, our results on K-ATPase can be discussed in relation to the well-characterized gastric H-K-ATPase (9$10,16)$.

$\mathrm{H}-\mathrm{K}-\mathrm{ATPase}$ belongs to the $\mathrm{E}_{1}-\mathrm{E}_{2}$ type of ATPases that are inhibited by vanadate (9). In the present study, K-ATPase activity was also inhibited by a low concentration $(20 \mu \mathrm{M})$ of vanadate. Although it has been suggested that gastric $\mathrm{H}-\mathrm{K}-$

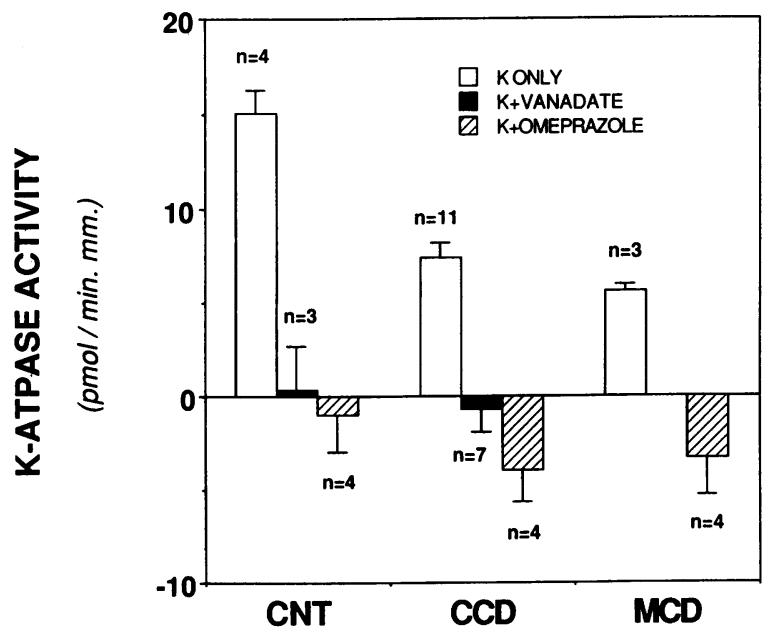

Figure 4. Effects of vanadate $(20 \mu \mathrm{M})$ and omeprazole $(100 \mu \mathrm{M})$ on $\mathrm{K}-\mathrm{ATP}$ Pase activity in the CNT, CCD, and MCD. Each bar is mean \pm SEM of $n$ animals. See results for explanation of the negative values. 


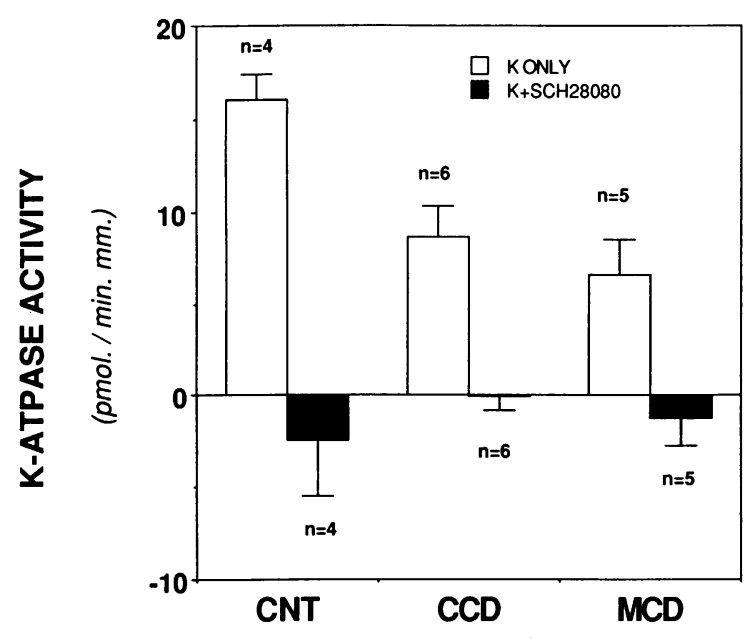

Figure 5. Effect of SCH $28080(100 \mu \mathrm{M})$ on K-ATPase activity in the $\mathrm{CNT}, \mathrm{CCD}$, and MCD. Each bar is mean \pm SEM of $n$ animals.

ATPase is a variant of the widely distributed Na-K-ATPase, the gastric enzyme is neither dependent on $\mathrm{Na}^{+}$nor is it inhibited by ouabain. In the present study, K-ATPase activity also was neither dependent on $\mathrm{Na}^{+}$nor was it inhibited by ouabain. These biochemical similarities suggest that K-ATPase in the CNT, CCD, and MCD has some properties of the well-characterized gastric H-K-ATPase $(8-10,16)$. Note that a K-activated ATPase, insensitive to ouabain and sensitive to inhibition by vanadate, has also been isolated from the brush border membranes of the rabbit descending colon epithelium (17). Furthermore, a vanadate-sensitive ATPase activity has also been reported to be present in the turtle bladder epithelial cells (18). The physiological functions of the vanadate-sensitive (and ouabain insensitive) ATPases in the kidney, descending colon, or turtle bladder have not been established.

In contrast to vanadate, omeprazole is a relatively specific inhibitor of gastric H-K-ATPase (10). In the present study, $\mathrm{K}-\mathrm{ATPase}$ activity in the CNT, CCD, and MCD was completely inhibited by $100 \mu \mathrm{M}$ omeprazole (Fig. 4). Omeprazole is a pro-drug that is converted to an active inhibitor of $\mathrm{H}-\mathrm{K}$ ATPase in the acidic environment of gastric canaliculi (19). The active form of omeprazole combines with sulfhydryl groups of gastric H-K-ATPase (20). Recently, a new compound, $\mathrm{SCH} 28080$, which does not need to be activated in the tissue, has been shown to inhibit both gastric acid secretion and H-K-ATPase $(11,21)$. Furthermore, it has been shown that SCH 28080 inhibits gastric H-K-ATPase by competing with $\mathrm{K}^{+}(11,12)$. Therefore, it seems that $\mathrm{SCH} 28080$ is a relatively more specific inhibitor of H-K-ATPase than even omeprazole. In the present study, we have demonstrated that K-ATPase activity in the CNT, CCD, and MCD was completely inhibited by SCH $28080(100 \mu \mathrm{M})$ (Fig. 5). Therefore, based on our studies with various inhibitors of gastric H-KATPase, the K-ATPase in the CNT, CCD, and MCD is probably similar to gastric H-K-ATPase.

Previous investigators have not been able to detect H-KATPase activity in the whole kidney preparations (22) probably because K-ATPase is present only in a few nephron segments (CNT, CCD, and MCD), and is a very small fraction of total ATPases along the whole nephron (13). Using a very sensitive ATPase microassay for individual nephron segments, we were able to detect K-ATPase activity in the CNT, CCD, and MCD. Doucet and Marsy (23) have now also reported an ouabain-insensitive (and vanadate sensitive) K-ATPase activity in the CNT, CCD, and MCD by using a different enzyme assay procedure. However, they have not studied the effect of SCH 28080 on K-ATPase in these segments.

$\mathrm{K}$-ATPase activity in the CNT was the highest among the three segments and was twice that of the CCD or MCD. However, when compared with Na-K-ATPase activity which we determined previously using a similar assay procedure (13), $\mathrm{K}$-ATPase activities in the CNT is only $14 \%$ of Na-K-ATPase activity in this segment. K-ATPase activities in the CCD and MCD were 29 and $46 \%$ of Na-K-ATPase activities, respectively, in these segments (13). Na-K-ATPase is known to be responsible for $\mathrm{Na}^{+}$reabsorption (and $\mathrm{K}^{+}$secretion), which occurs to a greater extent in the CNT and CCD than in the MCD. On the other hand, the physiological role of K-ATPase is not known at the present time. It is possible that it may be involved in $\mathrm{K}^{+}$reabsorption and/or $\mathrm{H}^{+}$secretion in these segments of the nephron. Note that K-ATPase is stimulated at very low concentrations of $\mathrm{K}^{+}(<1.0 \mathrm{mM})$, which is consistent with the physiological concentrations of $\mathrm{K}^{+}$in the tubular lumen of these segments. In addition, it has been shown that K-ATPase activity in the MCD is increased when the animals are given a low-K diet (reference 23, and our unpublished observations). If $\mathrm{K}^{+}$reabsorption in the $\mathrm{MCD}$ during $\mathrm{K}$ depletion is dependent on K-ATPase, the enzyme should be present in the apical membrane of K-absorbing cells in the MCD. However, this remains to be established.

Note that Na-K-ATPase activity in the MCD has also been shown to increase in animals fed a low-K diet (23-25). If Na-K-ATPase is responsible for $\mathrm{K}^{+}$conservation during $\mathrm{K}$ depletion by increasing $\mathrm{K}^{+}$reabsorption in the $\mathrm{MCD}$, this enzyme should be present in the apical membrane of K-absorbing cells in this segment. However, Na-K-ATPase has only been shown to be present in the basolateral membranes of the principal cells of CCD and MCD, and apical Na-K-ATPase has not been shown to be present either in principal or in intercalated cells in CCD or MCD (26). Therefore, the physiological significance of an increase in Na-K-ATPase activity in the MCD during $\mathrm{K}$ depletion is not known.

In summary, we have demonstrated that a K-ATPase, with some properties similar to gastric H-K-ATPase, is present in the CNT, CCD, and MCD. The physiological role of K-ATPase in these segments of the mammalian nephron remains to be determined.

\section{Acknowledgments}

We thank Judy Adams for typing the manuscript.

This work was supported by National Science Foundation grant PCB-8518000.

\section{References}

1. Giebisch, G., G. Malnic, and R. W. Berliner. 1986. Renal transport and control of potassium excretion. In The Kidney. B. M. Brenner and F. C. Rector, editors. W. B. Saunders Co., Philadelphia. 177-205.

2. Alpern, R. J., D. G. Warnock, and F. C. Rector. 1986. Renal acidification mechanisms. In The Kidney. B. M. Brenner and F. C. Rector, editors. W. B. Saunders Co., Philadelphia. 206-249. 
3. Stone, D. K., D. W. Seldin, J. P. Kokko, and H. R. Jacobson. 1983. Anion dependence of rabbit medullary collecting duct acidification. J. Clin. Invest. 71:1505-1508.

4. Gluck, S., and Q. Al-Awqati. 1984. An electrogenic protontranslocating ATPase from bovine kidney medulla. J. Clin. Invest. 73:1704-1710.

5. Garg, L. C., and N. Narang. 1985. Stimulation of an N-ethylmaleimide-sensitive ATPase in the collecting duct segments of the rat nephron by metabolic acidosis. Can. J. Physiol. Pharmacol. 63:12911296.

6. Kaunitz, J. D., R. D. Gunther, and G. Sachs. 1985. Characterization of an electrogenic ATP and chloride-dependent proton translocating pump from rat renal medulla. J. Biol. Chem. 260:11567-11573.

7. Narang, N., and L. C. Garg. 1987. Vanadate-sensitive (and ouabain-insensitive) ATPase activity in medullary segments of rat nephron. Kidney Int. 31:413. (Abstr.)

8. Garg, L. C., and N. Narang. 1987. Ouabain-insensitive K-ATPase activity in distal nephron segments of the rabbit. Fed. Proc. 46:363. (Abstr.)

9. Faller, L., R. Jackson, D. Malinowska, E. Mukidjam, E. Rabon, G. Saccomani, G. Sachs, and A. Smolka. 1982. Mechanistic aspects of gastric $\left(\mathrm{H}^{+}-\mathrm{K}^{+}\right)$-ATPase. Ann. NY Acad. Sci. 402:146-163.

10. Fellenius, E., T. Berglindh, G. Sachs, L. Olbe, B. Elander, S. Sjostrand, and B. Wallmark. 1981. Substituted benzimidazoles inhibit gastric acid secretion by blocking H-K-ATPase. Nature (Lond.). 290:159-161.

11. Wallmark, B., C. Briving, J. Fryklund, K. Munson, R. Jackson, J. Mendlein, E. Rabon, and G. Sachs. 1987. Inhibition of gastric $\mathrm{H}^{+}$, $\mathrm{K}^{+}$-ATPase is a $\mathrm{H}^{+}$-activated oxidizing agent of sulfhydryl groups. $J$. Biol. Chem. 260:4591-4597.

12. Scott, C. K., E. Sundell, and L. Castrovilly. 1987. Studies on the mechanism of action of the gastric microsomal $\left(\mathrm{H}^{+}-\mathrm{K}^{+}\right)$-ATPase inhibitors: SCH 32651 and SCH 28080. Biochem. Pharmacol. 36:97104.

13. Garg, L. C., M. A. Knepper, and M. B. Burg. 1981. Mineralocorticoid effects on Na-K-ATPase in individual nephron segments. Am. J. Physiol. 240:F536-F544.

14. Schoner, W., C. Von Ilberg, R. Kramer, and W. Seubert. 1967. On the mechanism of $\mathrm{Na}^{+}$and $\mathrm{K}^{+}$-stimulated hydrolysis of adenosine triphosphate. 1. Purification and properties of a $\mathrm{Na}^{+}$and $\mathrm{K}^{+}$activated ATPase from ox brain. Eur. J. Biochem. 1:334-363.
15. Ben Abdelkhalek, M., C. Barlet, and A. Doucet. 1986. Presence of extramitochondrial anion-stimulated ATPase in the rabbit kidney: localization along the nephron and the effect of corticosteroids. $J$. Membr. Biol. 89:225-240.

16. Wallmark, B., H. Larsson, and L. Humble. 1984. The relationship between gastric acid secretion and gastric H-K-ATPase activity. $J$. Biol. Chem. 260:13681-13684.

17. Gustin, M. C., and D. B. P. Goodman. 1981. Isolation of brush border membrane from the rabbit descending colon epithelium. Partial characterization of a unique K-activated ATPase. J. Biol. Chem. 256:10651-10656.

18. Youmouns, S. J., and W. A. Brodsky. 1987. Vanadate inhibition of $\mathrm{H}^{+}$transport in membrane vesicles from turtle bladder epithelial cells. Biochim. Biophys. Acta. 900:88-102.

19. Wallmark, B., A. Bradstrom, and H. Larsson. 1984. Evidence for acid-induced transformation of omeprazole into an active inhibitor of $\left(\mathrm{H}^{+}-\mathrm{K}^{+}\right)$ATPase within the parietal cells. Biochim. Biophys. Acta. 778:549-558.

20. Im, W. B., J. C. Sih, D. P. Blakeman, and J. P. McGrath. 1985. Omeprazole, a specific inhibitor of gastric $\mathrm{H}^{+}, \mathrm{K}^{+}$-ATPase is a $\mathrm{H}^{+}$-activated oxidizing agent of sulfhydryl groups. J. Biol. Chem. 260:45914597.

21. Long, J. F., P. J. S. Chin, M. J. Derelanko, and M. Steinberg. 1983. Gastric antisecretory and cytoprotective activities of $\mathrm{SCH}$ 28080. J. Pharmacol. Exp. Ther. 226:114-120.

22. Machen, T. E., and J. G. Forte. 1979. Gastric secretion. In Membrane Transport in Biology. G. Giebish, D. C. Tosteson, and H. H. Ussing, editors. Springer-Verlag New York Inc., New York. 4B:693-747.

23. Doucet, A., and S. Marsy. 1987. Characterization of K-ATPase activity in distal nephron: stimulation by potassium depletion. Am.J. Physiol. 253:F418-F423.

24. Hayashi, M., and A. I. Katz. 1987. The kidney in potassium depletion. I. Na-K-ATPase activity and $\left[{ }^{3} \mathrm{H}\right]$ ouabain binding in MCT. Am. J. Physiol. 252:F437-F446.

25. Imbert-Teboul, M., A. Doucet, S. Marsy, and S. Siaume-Perez. 1987. Alterations in enzymatic activities along rat collecting tubule in potassium depletion. Am. J. Physiol. 253:F408-F417.

26. Kashgarian, M., D. Biemesderfer, M. Caplan, and B. Forbush III. 1985. Monoclonal antibody to Na, K-ATPase: immunocytochemical localization along nephron segments. Kidney Int. 28:899-913. 